\title{
Localization capability and limitation of electron-multiplying charge-coupled, scientific complementary metal-oxide semiconductor, and charge-coupled devices for superresolution imaging
}

\author{
Tingwei Quan \\ Huazhong University of Science and Technology \\ Britton Chance Center for Biomedical Photonics \\ Wuhan National Laboratory for Optoelectronics \\ Wuhan 430074, China \\ and \\ Hubei University of Education \\ College of Mathematics and Economics \\ Wuhan 430205, China
}

\author{
Shaoqun Zeng \\ Zhen-Li Huang* \\ Huazhong University of Science and Technology \\ Britton Chance Center for Biomedical Photonics \\ Wuhan National Laboratory for Optoelectronics \\ Wuhan 430074, China
}

\begin{abstract}
Localization of a single fluorescent molecule is required in a number of superresolution imaging techniques for visualizing biological structures at cellular and subcellular levels. The localization capability and limitation of low-light detectors are critical for such a purpose. We present an updated evaluation on the performance of three typical low-light detectors, including a popular electron-multiplying CCD (EMCCD), a newly developed scientific CMOS (sCMOS), and a representative cooled CCD, for superresolution imaging. We find that under some experimental accessible conditions, the sCMOS camera shows a competitive and even better performance than the EMCCD camera, which has long been considered the detector of choice in the field of superresolution imaging. ( 2010 Society of Photo-Optical Instrumentation Engineers. [DOI: $10.1117 / 1.3505017$ ]
\end{abstract}

Keywords: single molecule localization; superresolution microscopy; low-light detector; noise model; maximum likelihood method.

Paper 10320RR received Jun. 8, 2010; revised manuscript received Aug. 27, 2010; accepted for publication Sep. 10, 2010; published online Nov. 19, 2010.

\section{Introduction}

Recently, a great breakthrough has been made in superresolution imaging for living cells. ${ }^{1-6}$ Localization-based superresolution microscopy, such as photoactivation localization microscopy ${ }^{4,5}$ (PALM) and stochastic optical reconstruction microscopy ${ }^{6}$ (STORM), is an intrinsically wide-field imaging approach and thus results in superior performances in live cell imaging. ${ }^{7,8}$ In this type of localization-based technique, a fraction of molecules is activated or switched on sparsely, such that the adjacent activated molecules normally are separated by more than the width of the point spread function, and could be distinguished through fluorescent imaging and localization. Repeating the activation and localization process enables precisely determining the positions of many molecules, a superresolution image could thus be reconstructed by overlaying these locations. ${ }^{8-10}$

Localization precision is usually defined as spatial resolution and has been detailed and extensively discussed. ${ }^{4,11-13}$ Generally, many factors influence the localization precision, and the performance of a detector is critical for such low-light imaging. In an early study by Ober et al., ${ }^{12}$ fundamental limits of the localization accuracy for a single molecule were fully analyzed by carefully considering both the photoactivatable molecule [in that case, green fluorescent protein (GFP)] and the optical system, including the emission rate of a single molecule, the efficiency of the optical system, the acquisition time, the effects of pixelation, and detector noise. However, this study was based on several popular CCD detectors available at that time.

\footnotetext{
*Address all correspondence to: Zhen-Li Huang, Britton Chance Center for Biomedical Photonics, Wuhan National Laboratory for Optoelectronics, Huazhong University of Science and Technology, Wuhan 430074, China; Tel: + 86-2787792033; Fax: +86-27-87792034; E-mail: leo@mail.hust.edu.cn.
}

Recently, another type of superior low-light detector, an electron-multiplying CCD (EMCCD) camera ${ }^{14,15}$ has become available and affordable, and thus has been used exclusively for many years in the field of superresolution imaging. ${ }^{10}$

Recently, a new type of high-performance low-light detector, the scientific CMOS (sCMOS) camera, was introduced and will be commercially available shortly. ${ }^{16}$ This detector is capable of simultaneously offering extremely low noise [e.g., a read noise of less than three electrons root mean square (RMS) at 100 full frames/s), rapid frame rates (e.g., 100 frames/s with a $2560 \times 2160$ array) and large field of view (5.5 megapixels, $6.5 \mu \mathrm{m}$ for each pixel). Therefore, it holds great potential for superresolution imaging. Moreover, with the rapid progresses in fluorescence labeling techniques, including the development of brighter fluorescent probes to increase signal and the standardized experimental protocols to minimize background noise, ${ }^{10,17}$ more detected photon signal could be experimentally obtainable and thus the superior role of an EMCCD camera in low-light imaging is being challenged. Obviously, an updated evaluation on the localization capacity and limitation of an EMCCD camera and the newly developed sCMOS camera will provide necessary guidance for the design of better superresolution imaging system.

Here we used a well-established approach, which is based on the combination of the point spread function (PSF) and a noise model, to quantify the performance of EMCCD and sCMOS cameras for superresolution imaging. A modified algorithm from the generalized gradient projection method was adopted to determine the position of a single fluorescent molecule. To

$1083-3668 / 2010 / 15(6) / 066005 / 6 / \$ 25.00$ (C) 2010 SPIE 
further understand the relation between the detector specification and the localization precision, discussions on the localization performance of a representative cooled CCD camera is included.

\section{PSF and Noise Model}

In this section, we employ the basic setup described by Ober et al., which consists of a single molecule at the focus of an objective lens and a planar detector. ${ }^{12}$ The PSF is introduced to model an individual fluorescent molecule and the conversion of optical signals to digital ones is derived for different detectors.

For isotropic fluorescent emission in an optical system without aberration and defocus, the spatial distribution of the emitted light intensity could be modeled ${ }^{18,19}$ by the PSF, which is given by

$$
\begin{aligned}
\operatorname{PSF}(x, y)= & \mid A \int_{0}^{1} J_{0}\left(k \frac { 1 } { M } \left[\left(x-M x_{p}\right)^{2}\right.\right. \\
& \left.\left.+\left(y-M y_{p}\right)^{2}\right]^{1 / 2} \mathrm{NA} \rho\right)\left.\rho d \rho\right|^{2},
\end{aligned}
$$

where $\left(x_{p}, y_{p}\right)$ is the actual position of the individual fluorescent molecule, NA is the numerical aperture of the objective lens, $M$ is the magnification of optical system, $k$ is the wave number, and $A$ is a constant amplitude.

Based on the working principles of the detectors, the optical signal is converted to electrical and digital signals eventually. Usually, an electron signal is considered equivalent to a digital signal because of their linear relation. ${ }^{15}$ In an image captured by the detectors, the number of photons $I_{i, j}$ collected in pixel $(i, j)$ can be written as

$$
I_{i, j}=n c \operatorname{PSF}(i, j),
$$

where $n$ is the total number of photons collected by the detector; $c$ is the normalizing scalar of PSF; $I_{i, j}$ converts to $q_{i, j}$ electrons in pixel $(i, j)$ with probability $P_{f}(i, j)$, which is given by

$$
P_{f}\left(I_{i, j}=q_{i, j}\right)=\frac{\exp \left(-\phi I_{i, j}\right)\left(\phi I_{i, j}\right)^{q_{i, j}}}{q_{i, j} !},
$$

where $\Phi$ is the quantum efficiency of the detector. For biological imaging applications, background noise usually results from autofluorescence and inactive fluorescent molecules, which can be modeled as Poisson noise. ${ }^{20}$ The intensity of background noise $I_{b}$ is converted to $s_{i, j}$ electrons in pixel $(i, j)$ with probability

$$
P_{b}\left(I_{b}=s_{i, j}\right)=\frac{\exp \left(-\phi I_{b}\right)\left(\phi I_{b}\right)^{s_{i, j}}}{s_{i, j} !} .
$$

According to Eqs. (3) and (4), the number of detected photons could be calculated for individual pixels. The corresponding digital signals are given by

$$
f_{i, j}=q_{i, j}+s_{i, j}+g .
$$

Here $g$ represents readout noise, which is considered as Gaussian noise. ${ }^{20}$ Note that Eq. (5) is valid for both sCMOS and CCD cameras.

However, extra electron-multiplying (EM) processes, which could be considered as Bernoulli processes, ${ }^{14}$ are involved in an EMCCD camera, thus Eq. (5) should be modified accordingly.
In the EM process, one electron is either converted into two electrons with probability $a$ or remains unchanged with probability 1- $a$. After multiplying $N$ times, the total EM gain value is $(1+a)^{N}$. Therefore, after the multiplication processes, the digital signal for an EMCCD camera is expressed by

$$
f_{i, j}=G\left(q_{i, j}+s_{i, j}, a, N\right)+g,
$$

where $G$ represents the electron multiplication process, and $g$ has the same meanings as in Eq. (5).

\section{Generalized Gradient Projection Method for Localizing Individual Fluorescent Molecule}

Several methods were proposed for the fluorescent molecule localization, including Gaussian fitting, ${ }^{13}$ FluoroBancroft, ${ }^{21}$ and maximum likelihood. ${ }^{7,12,19,22}$ In this paper, under the maximum likelihood framework, the generalized gradient projection method ${ }^{23,24}$ is used to localize an individual fluorescent molecule. Some constrained conditions were given to ensure convergence of the localization algorithm for signals with a low signal-to-noise ratio (SNR). For data generated from Eq. (5) or (6), the observed digital signal $f_{i, j}$ at pixel $(i, j)$ could be written with the following probability:

$$
P\left(I_{i, j}^{T}=f_{i, j}\right)=\frac{\left(I_{i, j}^{T}\right)^{f_{i, j}} \exp \left(-I_{i, j}^{T}\right)}{f_{i, j} !},
$$

where $I_{i, j}^{T}$ is the theoretical value of the sum of signal, background noise, and readout noise; $I_{i, j}^{T}$ is approached by ${ }^{25}$

$$
I_{i, j}^{T}=A \exp \left[-\frac{\left(i-x_{0}\right)^{2}+\left(j-y_{0}\right)^{2}}{2 \sigma^{2}}\right]+I_{b}+g .
$$

Here $\left(x_{0}, y_{0}\right)$ is the position of fluorescent molecule; and $A, I_{b}$, and $g$ denote the peak value of signal, the intensity of background noise, and the average readout noise, respectively. Note that all the parameters in Eq. (8) are intrinsically above zero. In addition, a joint probability describing the spatial distribution of the light intensity could be expressed by

$$
\prod_{i, j} P\left(I_{i, j}^{T}=f_{i, j}\right)
$$

The logarithm of Eq. (9) could be maximized by adjusting the parameters in Eq. (8). Based on the information described above, the nonlinear optimum problem (NOP) is acquired as

$$
\begin{aligned}
\min L= & \left(\sum_{1 \leq i, j \leq n} I_{i, j}^{T}\right)-\sum_{1 \leq i, j \leq n} f_{i, j} \log \left(I_{i, j}^{T}\right) \\
& \text { subject to } \mathrm{A}>0, \quad x_{0}>0, \\
& y_{0}>0, \quad \sigma>0, \quad I_{b}+g>0 .
\end{aligned}
$$

The generalized gradient projection method ${ }^{23,24}$ could be used to solve the NOP, and the optimal value of $\left(x_{0}, y_{0}\right)$ is obtained as the position of the fluorescent molecule.

\section{Results and Discussion}

In the following discussion, the specifications for a popular EMCCD camera ${ }^{26}$ (Andor iXon DU 897) and a representative 
(1) PSF, background noise and shot noise

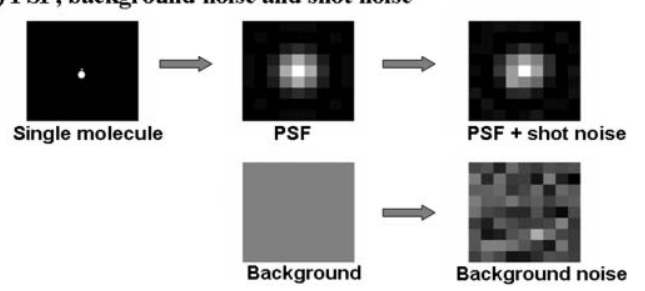

(2) Simulating image for $\mathrm{SCMOS}$ and CCD

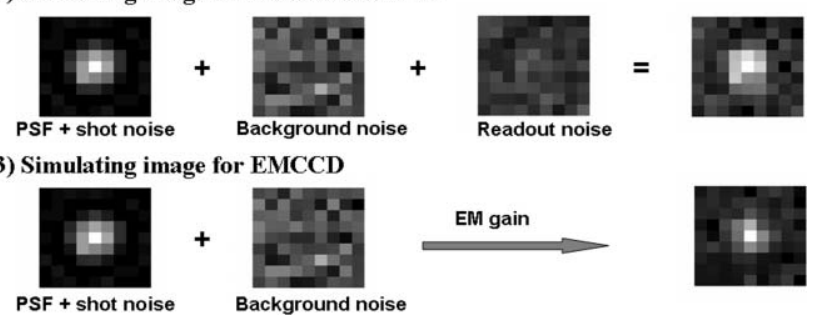

Fig. 1 PSF and noise models for the simulation.

cooled CCD camera ${ }^{27}$ (Photometrics CoolSNAP HQ ${ }^{2}$ ) are used. For the sCMOS camera, data were adopted from the white paper of sCMOS technology. ${ }^{16}$ The PSF and noise models used in the simulation are shown in Fig. 1. All of the simulations and calculations were carried out with MATLAB 7.0.

Noise performance, which is mainly a result of dark current and readout noise, is crucial for assessing the performance of a detector. ${ }^{25}$ In most cases, the dark current originates from the thermal noise of the detector and could be effectively reduced with decreased temperature. Therefore, for scientific detectors with proper cooling accessories, the major concern should be the readout noise rather than the dark current. Specifically, for sCMOS and CCD cameras, which usually work under low temperatures (typically $<-30^{\circ} \mathrm{C}$ ), the dark current is negligible. The readout noise is typically $2 e /$ pixel for the sCMOS camera ${ }^{16}$ and $4.5 \mathrm{e}$ /pixel for the CCD camera. ${ }^{27}$ As for the EMCCD camera, the effective elimination of readout noise could be realized by the electron multiplication process, as a result, additional noise is induced by the process itself. Effects from this additional noise should be quantified by an excess noise factor, thus a different digitization algorithm from SCMOS and CCD cameras should be introduced for a EMCCD camera [see Eqs. (5) and (6)]. In addition, note that the dark current is also negligible for an EMCCD camera, in which standard cooling accessories are typically installed.

In the pixelation process, it is important to select the proper parameters to analyze the localization precision of individual fluorescent molecule. Here, we do not simply use SNR as an essential factor critical for localization precision. Instead, for such a purpose it is necessary to use the following parameters: the background noise from the environment of the single molecule, the number of photons collected by the detector, and three important technical specifications from the detector (including quantum efficiency, EM gain, and readout noise). The reason is as follows. The noise performance is strongly dependent on the detector and thus has a different level of contribution to the corresponding SNR. In this sense, the SNR is not suitable to quantify the relation between localization precision and a detector. Nevertheless, all the former five parameters are independent of each other and all affect the SNR.
Table 1 Some important parameters for simulation.

\begin{tabular}{lllll}
\hline & & \multicolumn{3}{c}{ Value } \\
\cline { 3 - 5 } Parameter & Symbol & EMCCD & sCMOS & $\mathrm{CCD}$ \\
\hline Emission wavelength $(\mathrm{nm})$ & $\lambda_{\mathrm{em}}$ & 500 & 500 & 500 \\
Numerical aperture & $\mathrm{NA}$ & 1.4 & 1.4 & 1.4 \\
Pixel size $(\mu \mathrm{m})$ & $p$ & 16 & 6.5 & 6.45 \\
Quantum yield & $\phi$ & 0.9 & 0.6 & 0.6 \\
Readout noise $(e /$ pixel) & $g$ & 45 & 2 & 4.5 \\
\hline
\end{tabular}

Simulations were carried out using parameters that match a typical optical setup. Simulated images $(17 \times 7$ pixels $)$ of a single fluorescent molecule were generated from Eqs. (5) and (6) for the sCMOS (or CCD) and EMCCD cameras, respectively. The sizes of these images were large enough compared to the corresponding Airy disk. The position of an individual fluorescent molecule was randomly selected in the central pixel. The average localization precision for the detectors was then calculated according to Eq. (10). Some important parameters of the optical system are listed in Table 1. These parameters are appropriate for popular detectors and typical experimental conditions for low-light imaging and are applied for all simulations and calculations in the following, unless explicitly stated.

The localization precision was quantified as a function of EM gain value in the presence of background noise (see Fig. 2). Note that the magnification of the optical system was set to be 160. As shown in Fig. 2, the localization precision was kept nearly unchanged when the EM gain $\left(M_{g}\right)$ value is larger than 20. The result is probably due to the fact that the intensities of additional noise and readout noise are proportional to the factors $F=2-1 / M_{g}$ and $1 / M_{g}$, respectively. ${ }^{14}$ Thus, larger EM gain values only lead to slight changes of these factors and do not boost the localization precision. In the following analysis, the EM gain value was typically fixed to a representative value of

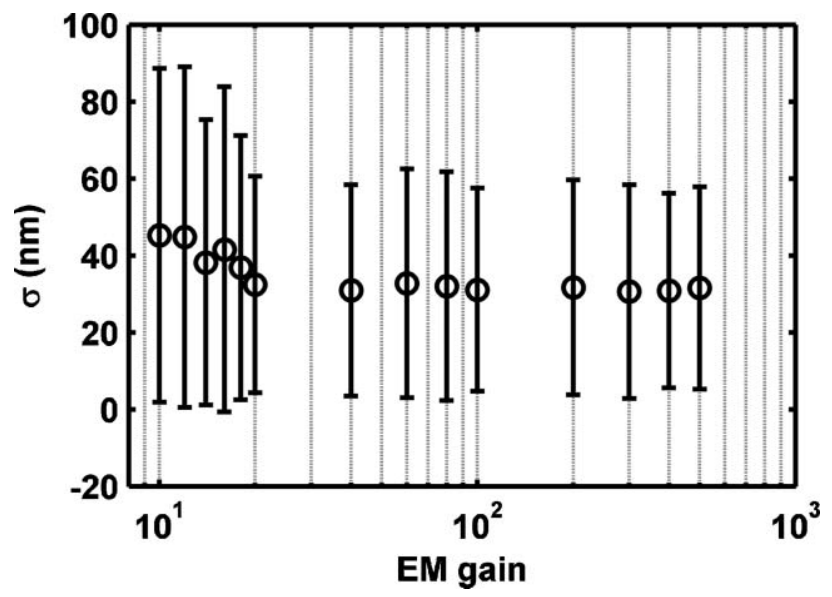

Fig. 2 Dependence of localization precision on the EM gain value of an EMCCD camera in the presence of background noise. The intensity of background noise was set to be $1 / 2$ of the peak value of the signal. The number of detected photons was set to be 100 . 
Quan, Zeng, and Huang: Localization capability and limitation of electron-multiplying ...
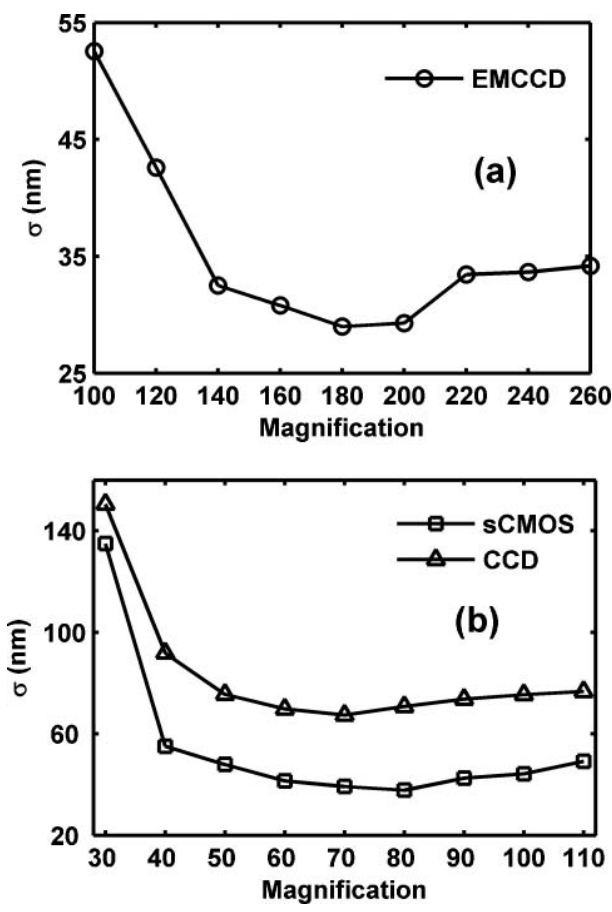

Fig. 3 Dependence of the localization precision on the optical system magnification for different detectors in the presence of background noise. The intensity of background noise and the number of detected photons are the same as those in Fig. 2.

100. Note that our finding is supported by Wu et al., ${ }^{28}$ who found that the EM gain in an EMCCD camera should be optimized to achieve the best localization precision.

The relation between the magnification of the optical system and the localization precision was calculated with simulated images and is shown in Fig. 3. It is found that the optimal magnification for maximizing localization precision is $\sim 180$ for EMCCD camera and $\sim 80$ for sCMOS and CCD cameras, respectively. Interestingly, this finding is supported by the following theoretical analysis. It is generally accepted that the magnification of the optical system should be adjusted to ensure that at least 9 to 16 pixels (or 3 to 4 pixels in each dimension) in the detector plane are used to cover the Airy disk, considering a trade-off between signal quality and the number of pixels used to collect photons. ${ }^{12,13}$ Since the diameter of Airy disk is 4 times of the width of the Gaussian kernel in the detector plane, the desirable ratio between the number of pixels and the number of Gaussian kernels is 0.75 to 1 . It was reported that the width of Gaussian kernel in the detector plane approximately equals to $0.21 \lambda M$ /numerical aperture ${ }^{25}(\mathrm{NA})$. Using the parameters in Table 1, the optimal magnification of the optical system could be calculated to be about $M=70$ to 80 for both SCMOS and CCD cameras and $M=160$ to 200 for an EMCCD camera.

The localization performance of the detectors under representative signal and background noise levels is shown in Fig. 4. It is found that, for a noisy image where the detected photons are heavily insufficient $(<300)$ and the scattered photons or background fluorescence level are relatively high (for example, $A / I_{b}=2$ ), EMCCD camera shows superior performance on the localization precision compared to both sCMOS and CCD cameras. However, when the number of detected photons
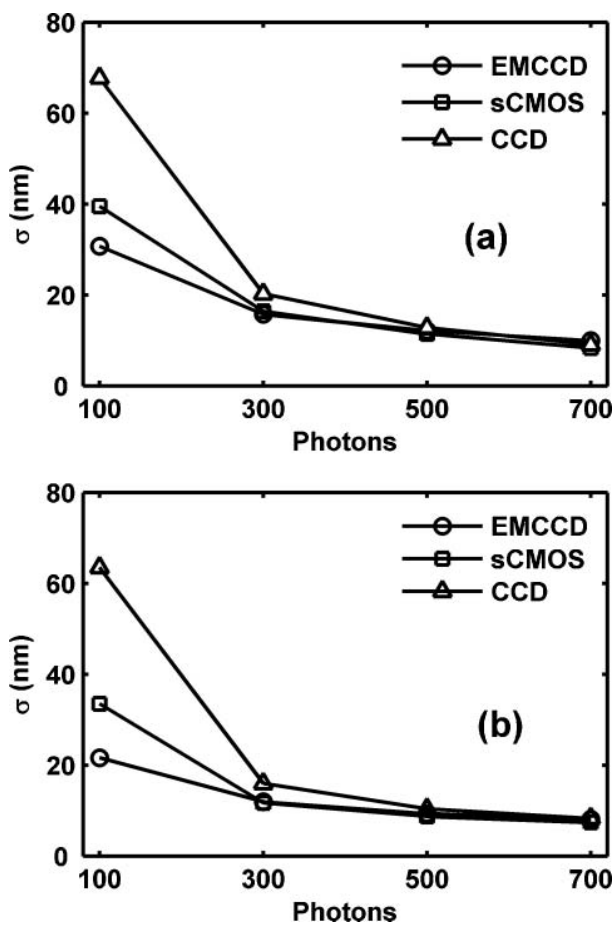

Fig. 4 Dependence of the localization precision on the number of detected photons $(A)$ and background noise levels: $\left(I_{b}\right)$. (a) $A / I_{b}=2$ and (b) $A / I_{b}=4.5$.

exceeds 300, only a slight difference could be found in localization performance between SCMOS and EMCCD cameras. Note that when the total number of detected photons is 100 and $A / I_{b}$ is 2 , the number of signal photons is about 21 photons in the peak pixel and the background noise level is 11 photons/pixel. As the localization precision decreases rapidly due to the low SNR in the image, detecting $<100$ photons/molecule in a strong background, i.e., $A / I_{b}<2$, will not be practical for all of these detectors.

It is interesting to evaluate when to use sCMOS camera and even a cheaper detector, CCD camera, for superresolution imaging. Here we chose $50 \mathrm{~nm}$ as the desired localization precision, which is acceptable for monitoring dynamic process in living cells. It is found that, when the total number of detected photons is 300 and $A / I_{b}$ is 2 (corresponding to $\sim 60$ signal photons in the peak pixel and 30 photons/pixel in the background noise level), no notable difference exists in the localization precision for the detectors studied [Fig. 5(a)]. More interestingly, both the sCMOS and CCD cameras could work highly competitively under fewer detected photons, if the signal-to-background ratio value is made larger [i.e., $A / I_{b}=4.5$, see Fig. 5(b)]. This is experimentally accessible with the combination of using bright fluorescent probes (such as mEosFP and $\mathrm{Cy} 5$, where hundreds of photons could be detected within a 10-ms time interval ${ }^{29-31}$ ) and minimizing background photons (i.e., collected scattered photons and background fluorescence) from proper experimental procedures. ${ }^{10}$ This finding indicates that, with the development of brighter fluorescent probes, an sCMOS camera would boost its potential application in superresolution imaging of living cells by offering simultaneously both a large field of view and a high image acquisition speed. 

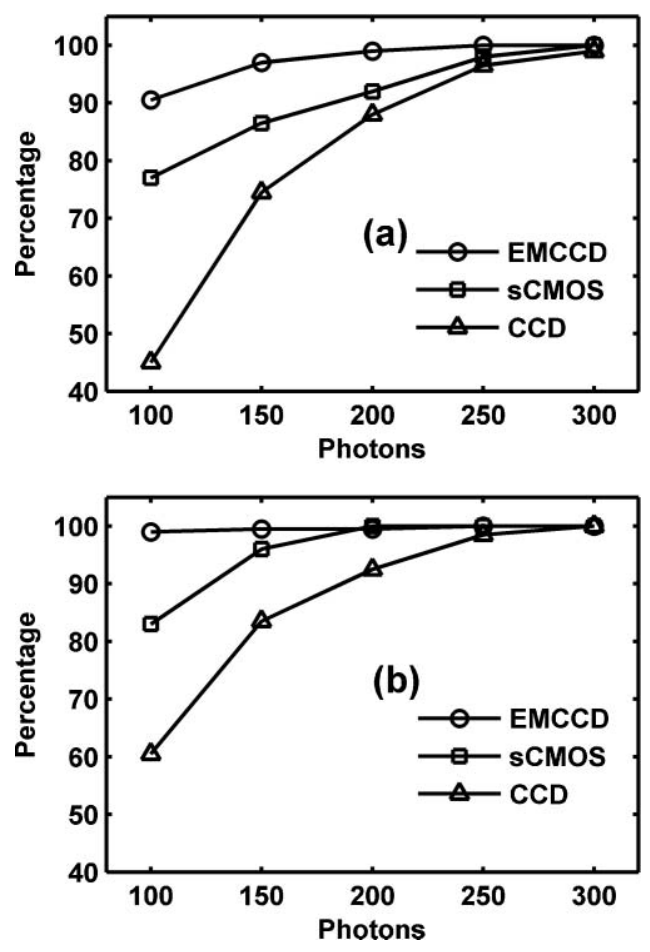

Fig. 5 Relation between number of collected photons and the percentage of localization precision better than $50 \mathrm{~nm} ; A / I_{b}$ was set to be 2 in (a) and 4.5 in (b), respectively.

Calculations based on the method reported previously in the literature were also performed to further analyze the localization performance of the detectors. For sCMOS and CCD cameras, the localization precision for individual fluorescent molecule could be rewritten as ${ }^{13}$

$$
\left\langle\Delta x^{2}\right\rangle=\frac{s^{2}+a^{2} / 12}{\phi N}+\frac{8 \pi s^{4}\left(\phi I_{b}+N_{r}^{2}\right)}{a^{2}(\phi N)^{2}} .
$$

For an EMCCD camera, Eq. (11) must be modified to reflect the electron multiplication process:

$$
\left\langle\Delta x^{2}\right\rangle=\frac{2 s^{2}+a^{2} / 12}{\phi N}+\frac{8 \pi s^{4}\left(\phi I_{b}\right)}{a^{2}(\phi N)^{2}},
$$

where $s$ is the width of Gaussian kernel, $a$ is the pixel size, $I_{b}$ is the background noise, $N_{r}$ is the readout noise, $\phi$ is the quantum efficiency, and $N$ is the number of the photons collected (see the appendix).

Calculation from Eqs. (11) and (12) suggests that no notable difference in the localization precision exits between sCMOS and EMCCD cameras when the number of detected photons is more than 300 (Fig. 6). However, the localization precision calculated from Eqs. (11) and (12) is obviously overestimated (see data for 100 detected photons in Figs. 4 and 6). This may be attributed to the difference between the theoretic optimal position and the actual position of an individual fluorescent molecule, since this difference is neglected in the theoretic analysis. ${ }^{13}$ This finding indicates that the results from the simulation match better with real experimental phenomena than those from the theoretical calculation.
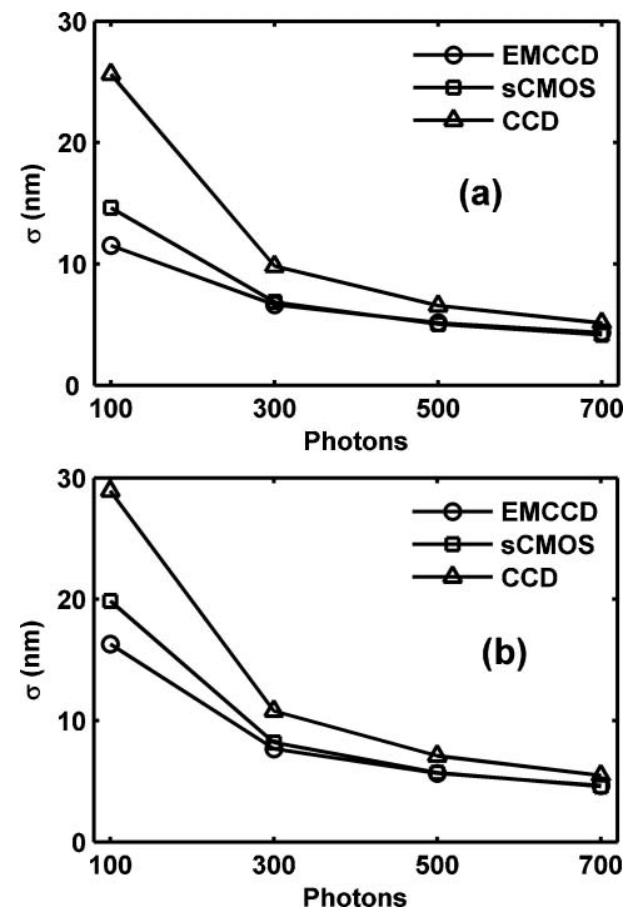

Fig. 6 Theoretical localization precision (a) without and (b) with background noise of 11 photons/pixel $\left(I_{b}=11\right)$. Other parameters were set to be the same or comparable to those used previously.

\section{Conclusion}

We studied the localization capability and limitation of three typically low-light detectors (sCMOS, EMCCD, and CCD cameras) for superresolution imaging. The PSF and noise model were introduced to convert the optical signals into digital ones. The generalized gradient projection method was used to localize an individual fluorescent molecule detected by the detectors. We found that the newly developed sCMOS camera could show competitive performance with the EMCCD camera when better fluorescence labeling techniques are used.

\section{Appendix}

By employing procedures similar to those reported by Thompson et al., ${ }^{13}$ the equation for calculating the localization precision for an EMCCD camera is derived as follows. Based on the criterion of least-squares fitting, the sum of squared errors is written as

$$
\chi^{2}(x)=\sum \frac{\left[y_{i}-N_{i}(x)\right]^{2}}{\sigma_{i}^{2}} .
$$

Here, $y_{i}$ is the observed number of photons, $N_{i}(x)$ is the expectation of number of photons from a molecule located at $x$, and $\sigma_{i}^{2}$ is the intensity of noise. According to the Eq. (13), the expected error of the actual position $x_{0}$ in the $x$ direction is given by ${ }^{13}$

$$
\left\langle\Delta x^{2}\right\rangle=1 / \sum\left(N_{i}^{\prime 2} / \sigma_{i}^{2}\right)
$$

where $N_{i}$ could be approached by

$$
N_{i}=\frac{\phi N}{\sqrt{2 \pi} s} \exp \left(-\frac{\left(i-x_{0}\right)^{2}}{2 s^{2}}\right),
$$


where $N$ is the number of collective photons, $\Phi$ is the quantum efficiency, $x_{0}$ being the actual position of the molecule, and $s$ is the standard deviation of the Gaussian function. Considering the shot noise and addition noise in an EMCCD framework, the intensity of noise could be shown by ${ }^{14}$

$$
\sigma_{i}^{2}=(2-1 / M) N_{i} \approx 2 N_{i},
$$

where $M$ is the EM gain value (usually >100). Based on Eqs. (15) and (16), the partial expression on the right-hand side of Eq. (14) could be rewritten as

$$
N_{i}^{\prime 2} / \sigma_{i}^{2}=\frac{\phi N}{2 \sqrt{2 \pi} s} \exp \left[-\frac{\left(i-x_{0}\right)^{2}}{2 s^{2}}\right] \frac{\left(i-x_{0}\right)^{2}}{s^{4}} .
$$

Considering the characteristic of the Gaussian function, it is obvious that

$$
\sum \frac{1}{\sqrt{2 \pi} s} \exp \left(-\frac{\left(i-x_{0}\right)^{2}}{2 s^{2}}\right)\left(i-x_{0}\right)^{2}=s^{2} .
$$

According to Eqs. (14), (17), and (18), when only the shot noise and addition noise are presented in an EMCCD camera, Eq. (14) could be converted to

$$
\left\langle\Delta x^{2}\right\rangle=\frac{2 s^{2}}{\phi N}
$$

If background noise is also presented, using the procedures similar to those reported by Thompson et al., ${ }^{13}$ Eq. (19) is modified to

$$
\left\langle\Delta x^{2}\right\rangle=\frac{2 s^{2}+a^{2} / 12}{\phi N}+\frac{8 \pi s^{4}\left(\phi I_{b}\right)}{a^{2}(\phi N)^{2}} .
$$

\section{Acknowledgments}

This work was partially funded by the National Natural Science Foundation of China (Grant Nos. 30970691, 30927001, and 30925013), the Scientific Research Foundation for the Returned Overseas Chinese Scholars, Ministry of Education of China, Wuhan National Laboratory for Optoelectronics, and the Open Research Fund of State Key Laboratory of Bioelectronics, Southeast University.

\section{References}

1. S. W. Hell and J. Wichmann, "Breaking the diffraction resolution limit by stimulated-emission: stimulated-emission-depletion fluorescence microscopy," Opt. Lett. 19, 780-782 (1994).

2. A. Yildiz, J. N. Forkey, S. A. McKinney, T. Ha, Y. E. Goldman, and P. R. Selvin, "Myosin v walks hand-over-hand: single fluorophore imaging with 1.5-nm localization," Science 300, 2061-2065 (2003).

3. M. G. L. Gustafsson, "Nonlinear structured-illumination microscopy: wide-field fluorescence imaging with theoretically unlimited resolution," Proc. Nat. Acad. Sci. U.S.A. 102, 13081-13086 (2005).

4. S. T. Hess, T. P. K. Girirajan, and M. D. Mason, "Ultra-high resolution imaging by fluorescence photoactivation localization microscopy," Biophys. J. 91, 4258-4272 (2006).

5. E. Betzig, G. H. Patterson, R. Sougrat, O. W. Lindwasser, S. Olenych, J. S. Bonifacino, M. W. Davidson, J. Lippincott-Schwartz, and H. F. Hess, "Imaging intracellular fluorescent proteins at nanometer resolution," Science 313, 1642-1645 (2006).
6. M. J. Rust, M. Bates, and X. W. Zhuang, "Sub-diffraction-limit imaging by stochastic optical reconstruction microscopy (storm)," Nature Meth. 3, 793-795 (2006).

7. T. W. Quan, P. C. Li, F. Long, S. Q. Zeng, Q. M. Luo, P. N. Hedde, G. U. Nienhaus, and Z. L. Huang, "Ultra-fast, high-precision image analysis for localization-based super resolution microscopy," Opt. Express 18, 11867-11876 (2010)

8. B. Huang, M. Bates, and X. W. Zhuang, "Super-resolution fluorescence microscopy," Ann. Rev. Biochem. 78, 993-1016 (2009).

9. S. W. Hell, "Far-field optical nanoscopy," Science 316, 1153-1158 (2007).

10. T. J. Gould, V. V. Verkhusha, and S. T. Hess, "Imaging biological structures with fluorescence photoactivation localization microscopy," Nature Protoc. 4, 291-308 (2009).

11. A. R. Small, "Theoretical limits on errors and acquisition rates in localizing switchable fluorophores," Biophys. J. 96, L16-L18 (2009).

12. R. J. Ober, S. Ram, and E. S. Ward, "Localization accuracy in singlemolecule microscopy," Biophys. J. 86, 1185-1200 (2004).

13. R. E. Thompson, D. R. Larson, and W. W. Webb, "Precise nanometer localization analysis for individual fluorescent probes," Biophys. J. 82, 2775-2783 (2002).

14. M. S. Robbins and B. J. Hadwen, "The noise performance of electron multiplying charge-coupled devices," IEEE Trans. Electron Devices $\mathbf{5 0}$, 1227-1232 (2003).

15. M. J. DeWeert, J. B. Cole, A. W. Sparks and A. Acker, "Photon transfer methods and results for electron multiplication ccds," Proc. SPIE $\mathbf{5 5 5 8}$ 248-259 (2004).

16. http://www.scmos.com.

17. S. A. McKinney, C. S. Murphy, K. L. Hazelwood, M. W. Davidson, and L. L. Looger, "A bright and photostable photoconvertible fluorescent protein," Nature Meth. 6, 131-133 (2009).

18. A. Small, I. Ilev, V. Chernomordik, and A. Gandjbakhche, "Enhancing diffraction-limited images using properties of the point spread function," Opt. Express 14, 3193-3203 (2006)

19. F. Aguet, D. Van De Ville, and M. Unser, "A maximum-likelihood formalism for sub-resolution axial localization of fluorescent nanoparticles," Opt. Express 13, 10503-10522 (2005).

20. D. L. Snyder, A. M. Hammoud, and R. L. White, "Image recovery from data acquired with a charge-coupled-device camera," J. Opt. Soc. Am. A 10, 1014-1023 (1993).

21. S. B. Andersson, "Localization of a fluorescent source without numerical fitting," Opt, Express 16, 18714-18724 (2008).

22. C. S. Smith, N. Joseph, B. Rieger, and K. A. Lidke, "Fast, singlemolecule localization that achieves theoretically minimum uncertainty," Nature Meth. 7, 373-375 (2010).

23. Z. Gao, Y. Lai, and Z. Hu, "A generalized gradient projection method for optimization problems with equality and inequality constraints about arbitrary initial point," Acta Appl. Math. Sin. 12, 40-49 (1996).

24. D. Mayne and E. Polak, "Feasible directions algorithms for optimization problems with equality and inequality constraints," Math. Program. 11, 67-80 (1976)

25. B. Zhang, J. Zerubia, and J. Olivo-Marin, "Gaussian approximations of fluorescence microscope point-spread function models," Appl. Opt. 46, 1819-1829 (2007).

26. http://www.andor.com/pdfs/specs/L897SS.pdf.

27. http://www.photomet.com/products/datasheets/HQ2.pdf.

28. P. H. Wu, N. Nelson, and Y. Tseng, "A general method for improving spatial resolution by optimization of electron multiplication in ccd imaging," Opt. Express 18, 5199-5212 (2010).

29. F. Jonathan and G. Zheng, "Activatable smart probes for molecular optical imaging and therapy," J. Innov. Opt. Health Sci. 1, 45-61 (2008).

30. H. Bock, C. Geisler, C. A. Wurm, C. Von Middendorff, S. Jakobs, A. Schonle, A. Egner, S. W. Hell, and C. Eggeling, "Two-color far-field fluorescence nanoscopy based on photoswitchable emitters," Appl. Phys. B 88, 161-165 (2007).

31. J. Wiedenmann, S. Ivanchenko, F. Oswald, F. Schmitt, C. Rocker, A. Salih, K. D. Spindler, and G. U. Nienhaus, "EosFP, a fluorescent marker protein with UV-inducible green-to-red fluorescence conversion," Proc. Nat. Acad. Sci. U.S.A. 101, 15905-15910 (2004). 